\title{
Complement and tissue factor-enriched neutrophil extracellular traps are key drivers in COVID-19 immunothrombosis
}

\author{
Panagiotis Skendros, ${ }^{1,2}$ Alexandros Mitsios, ${ }^{2}$ Akrivi Chrysanthopoulou, ${ }^{2}$ Dimitrios C. Mastellos, ${ }^{3}$ Simeon Metallidis, ${ }^{4}$ \\ Petros Rafailidis, ${ }^{5}$ Maria Ntinopoulou, ${ }^{2}$ Eleni Sertaridou, ${ }^{6}$ Victoria Tsironidou, ${ }^{2}$ Christina Tsigalou, ${ }^{7}$ Maria Tektonidou, ${ }^{8}$ \\ Theocharis Konstantinidis, ${ }^{2}$ Charalampos Papagoras, ${ }^{1,2}$ Ioannis Mitroulis, ${ }^{1,2}$ Georgios Germanidis, ${ }^{4}$ John D. Lambris, ${ }^{9}$ \\ and Konstantinos Ritis ${ }^{1,2}$
}

'First Department of Internal Medicine, University Hospital of Alexandroupolis, and 'Laboratory of Molecular Hematology, Department of Medicine, Democritus University of Thrace, Alexandroupolis, Greece. ${ }^{3}$ National Center for Scientific Research 'Demokritos', Aghia Paraskevi, Athens, Greece. ${ }^{4}$ First Department of Internal Medicine, AHEPA University Hospital, Aristotle University of Thessaloniki, Thessaloniki, Greece. ${ }^{5}$ Second Department of Internal Medicine, University Hospital of Alexandroupolis, Democritus University of Thrace, Alexandroupolis, Greece. ${ }^{6}$ Intensive Care Unit, University Hospital of Alexandroupolis, Alexandroupolis, Greece. 'Laboratory of Microbiology, University Hospital of Alexandroupolis, Democritus University of Thrace, Alexandroupolis, Greece. ${ }^{8}$ First Department of Propaedeutic Internal Medicine, National and Kapodistrian University of Athens, Greece. ${ }^{9}$ Department of Pathology \& Laboratory Medicine, Perelman School of Medicine, University of Pennsylvania, Philadelphia, Pennsylvania, USA.

\begin{abstract}
Emerging data indicate that complement and neutrophils contribute to the maladaptive immune response that fuels hyperinflammation and thrombotic microangiopathy, thereby increasing coronavirus 2019 (COVID-19) mortality. Here, we investigated how complement interacts with the platelet/neutrophil extracellular traps (NETs)/thrombin axis, using COVID-19 specimens, cell-based inhibition studies, and NET/human aortic endothelial cell (HAEC) cocultures. Increased plasma levels of NETs, tissue factor (TF) activity, and sC5b-9 were detected in patients. Neutrophils of patients yielded high TF expression and released NETs carrying active TF. Treatment of control neutrophils with COVID-19 platelet-rich plasma generated TFbearing NETs that induced thrombotic activity of HAECs. Thrombin or NETosis inhibition or C5aR1 blockade attenuated platelet-mediated NET-driven thrombogenicity. COVID-19 serum induced complement activation in vitro, consistent with high complement activity in clinical samples. Complement C3 inhibition with compstatin Cp40 disrupted TF expression in neutrophils. In conclusion, we provide a mechanistic basis for a pivotal role of complement and NETs in COVID-19 immunothrombosis. This study supports strategies against severe acute respiratory syndrome coronavirus 2 that exploit complement or NETosis inhibition.
\end{abstract}

\section{Introduction}

Accumulated clinical evidence during the evolving coronavirus 2019 (COVID-19) pandemic indicates that severe acute respiratory syndrome coronavirus 2 (SARS-CoV-2) infection triggers thrombotic complications that affect multiple vital organs, increasing the mortality burden in patients with COVID-19 $(1,2)$

\section{Related Commentary: p. 5674}

Authorship note: PS, AM, and AC contributed equally to this work. JDL and KR are co-senior authors.

Conflict of interest: JDL is the founder of Amyndas Pharmaceuticals, which develops complement inhibitors for therapeutic purposes; he has a broad portfolio of patents describing the use of complement inhibitors for therapeutic purposes (www.lambris.com/ patents), some of which are developed by Amyndas (US patents 8946145/9371365, 9630992) and Apellis (US patents 6319897, 7989589, 7888323). JDL is also the inventor of the compstatin technology licensed to Apellis Pharmaceuticals (i.e., 4(1MeW)7W/ POT-4/APL-1 and PEGylated derivatives such as pegcetacoplan and APL-9). Copyright: (5) 2020, American Society for Clinical Investigation. Submitted: June 15, 2020; Accepted: August 5, 2020; Published: October 19, 2020 Reference information: / Clin Invest. 2020;130(11):6151-6157. https://doi.org/10.1172/JCl141374.
The pathological process of coagulopathy in COVID-19 is commonly characterized as an immunothrombosis, since it is related to a maladaptive host immune response fueled by excessive activation of innate immune pathways, deregulated thromboinflammation, and endothelial dysfunction $(1,2)$. Understanding the immunothrombotic mechanisms in COVID-19 constitutes a significant medical challenge.

Increased neutrophil counts have been associated with disease severity and poor prognosis in COVID-19, and extensive neutrophil infiltration of pulmonary capillaries has been described in autopsy specimens (3-7). In several inflammatory disorders (8), neutrophil extracellular traps (NETs) have been shown to exert thrombogenic activity through the expression of functionally active tissue factor (TF). NETs appear to be involved in COVID-19 as well (9).

Complement activation has been implicated as driver of the maladaptive inflammatory response in COVID-19. Complement can enhance neutrophil/monocyte activation and recruitment to the infected lungs, and several complement effectors, acting in concert with platelets, can fuel thromboinflammation, microvascular thrombosis, and endothelial dysfunction (thrombotic microangiopathy) $(7,10,11)$. Therefore, early clinical data have 
A

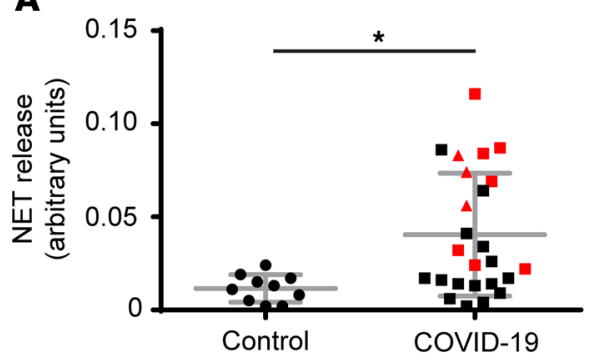

B

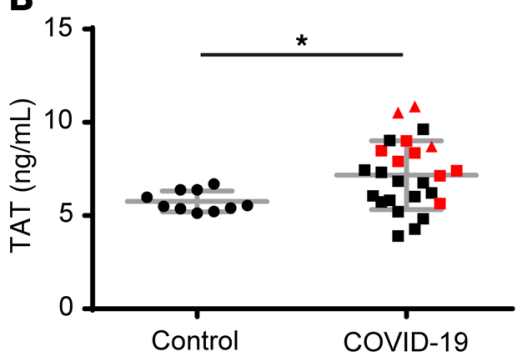

COVID-19

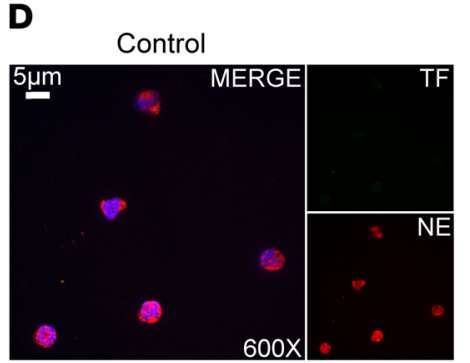

E

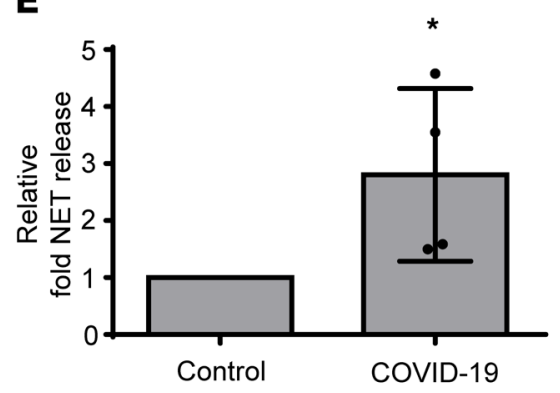

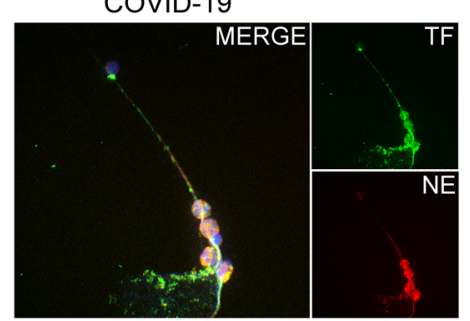

$\mathbf{F}$

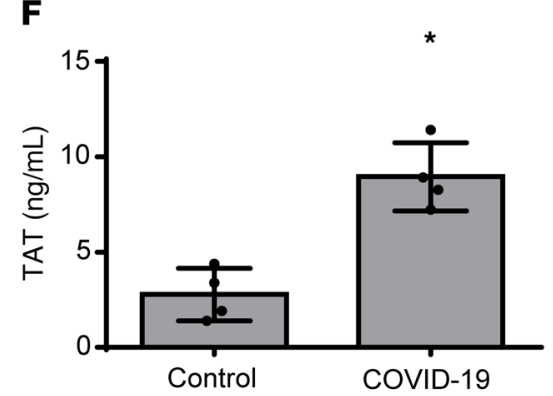

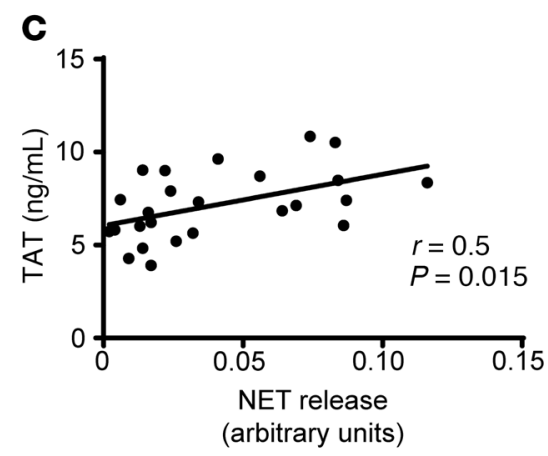

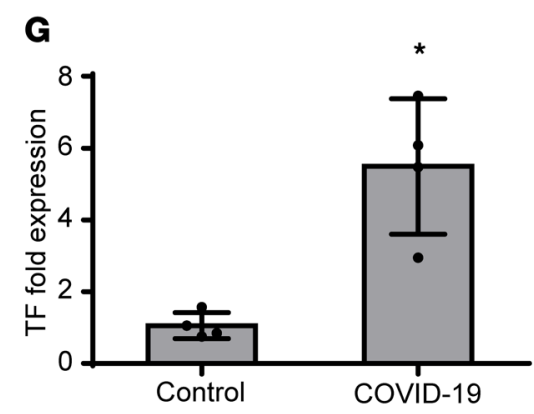

Figure 1. NETs in the coagulopathy of CoVID-19. (A) Myeloperoxidase (MPO)/DNA complex levels representing NET release in plasma from healthy individuals (controls, $n=10)$ and patients with COVID-19 $(n=25)$. (B) Thrombin-antithrombin (TAT) complex levels in plasma from controls and patients with COVID-19. In A and B, red squares indicate severe patients; red triangles, critical patients. (C) Correlation between MPO-DNA levels and TAT levels in patients with COVID-19. (D) Confocal fluorescence microscopy showing TF/neutrophil elastase (NE) staining in control and COVID-19 neutrophils. A representative example of 4 independent experiments is shown. Original magnification: $\times 600$; scale bar: $5 \mu$ m. Blue: DAPI; green: TF; red: NE. (E) MPO-DNA levels in NETs isolated from controls and patients with COVID-19. Data are from 4 independent experiments (mean \pm SD). (F) TAT levels in plasma from controls treated with NETs isolated from controls and patients with COVID-19. Data are from 4 independent experiments (mean \pm SD). (G) Fold expression of TF mRNA in neutrophils isolated from controls and patients with COVID-19. Data are from 4 independent experiments (mean \pm SD). D-G were performed in the same 4 patients (identified by an asterisk in Supplemental Table 1). All conditions were compared with controls. ${ }^{*}$ Statistical significance is $P<0.05$. A, B: Student's $t$ test; C: Spearman's correlation test; E-G: Friedman's test.

prompted the initiation of trials to evaluate various complement therapeutics in patients with COVID-19 (12).

The well-established crosstalk between complement and neutrophils in human immunothrombosis (13-15) led us to hypothesize that the collaboration of these innate immune systems may mediate early events leading to coagulopathy in COVID-19. Here, we investigated the role of neutrophils in COVID-19 thromboinflammation and provide evidence that complement activation potentiates the platelet/NETs/TF/thrombin axis during SARS-CoV-2 infection.

\section{Results and Discussion}

NETs are associated with activation of the TF/thrombin axis in COVID-19. We first used ELISA to measure the levels of myeloperoxidase (MPO)/DNA complexes (Figure 1A), a well-defined surrogate marker of NETosis (8), in the plasma of control patients and patients with COVID-19 and detected significantly increased levels in patients with COVID-19. The levels of these complexes were positively correlated with thrombin-antithrombin (TAT) activity, indicating activation of the $\mathrm{TF} /$ thrombin axis (Figure $1, \mathrm{~B}$ and $\mathrm{C}$ ). These findings were further confirmed by confocal immunofluorescence microscopy in neutrophils collected from 4 patients with severe COVID-19 (Supplemental Table 1; supplemental material available online with this article; https://doi. org/10.1172/JCI141374DS1). We found spontaneous formation of NETs expressing TF (Figure 1, D and E) that were functional, as indicated by TAT assay (Figure $1 \mathrm{~F}$ ). Moreover, the concomitant increase in TF mRNA levels (Figure 1G) further confirmed previous studies indicating that neutrophils constitute a source of TF in thromboinflammation. Although detection of NETosis markers in plasma by similar laboratory techniques has already been reported (9), we now show that NET release is positively correlated with in vivo thrombotic potency in COVID-19 (Figure 1C). 

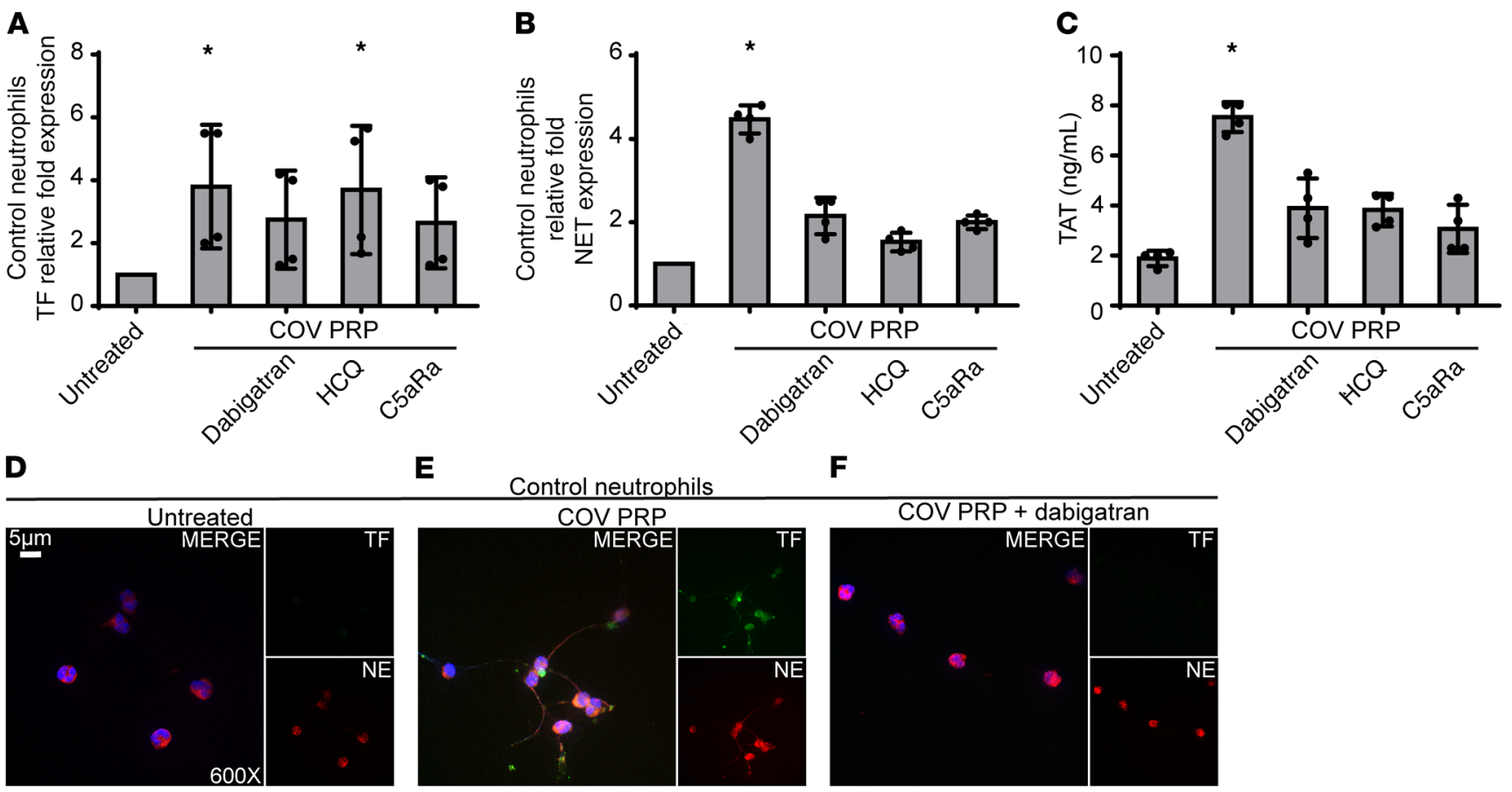

E Control neutrophils

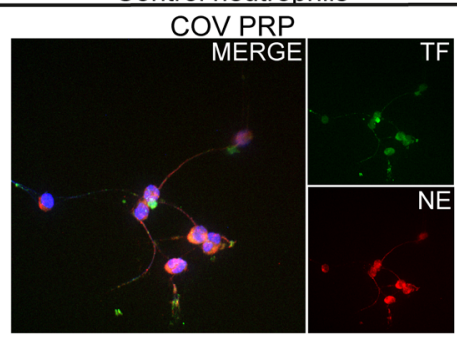

$\mathbf{F}$
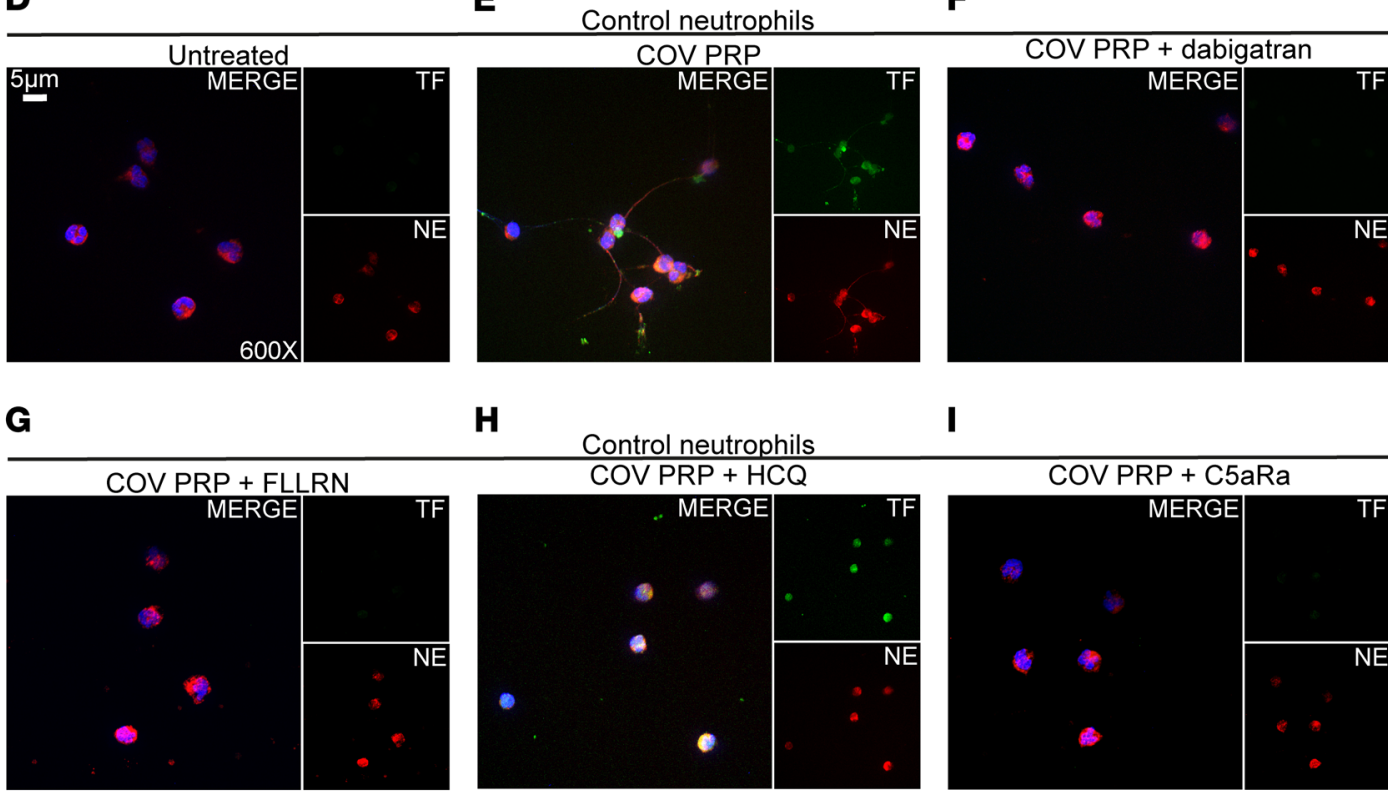

H

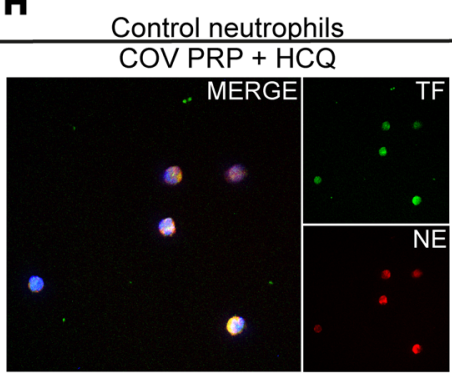

I

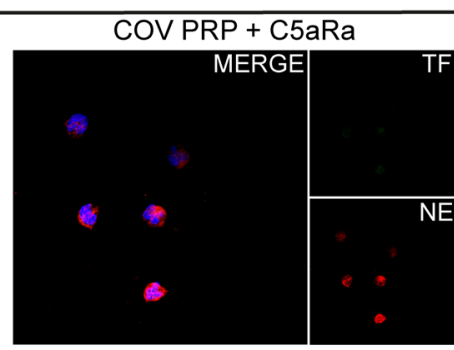

Figure 2. Inhibition of PRP-neutrophil interactions in COVID-19. (A) Relative fold expression of TF mRNA in control neutrophils treated with COVID-19derived PRP (COV PRP) and inhibited with dabigatran (thrombin inhibitor), HCQ (autophagy inhibitor), or C5aR1 antagonist (C5aRa/PMX-53). Data are from 4 independent experiments (mean \pm SD). (B) MPO-DNA complex levels in NETs isolated from control neutrophils treated with COV PRP and inhibited with dabigatran, HCQ, or C5aRa/PMX-53. Data are from 4 independent experiments (mean \pm SD). (C) TAT complex levels in control plasma stimulated with NET structures isolated from control neutrophils treated with COV PRP and inhibited with dabigatran, HCQ, or C5aR. Data are from 4 independent experiments (mean \pm SD). ( $\mathbf{D}$ and $\mathbf{E}$ ) Confocal fluorescence microscopy showing TF/neutrophil elastase (NE) staining in control neutrophils treated with COV PRP and inhibited with (F) dabigatran, (C) FLLRN (PAR1 receptor inhibitor), (H) HCQ, or (I) C5aRa/PMX-53. A representative example of 4 independent experiments is shown. Original magnification: $\times 600$; scale bar: $5 \mu \mathrm{m}$. Blue: DAPI, green: TF, red: NE. All conditions were compared with control/untreated. *Statistical significance at $P<0.05$. A-C: Friedman's test. The in vitro experiments mentioned above are also summarized in Supplemental Table 2. Conditions of realtime RT-PCR are described in Supplemental Table 3.

In an effort to corroborate our ex vivo findings, and because platelet-neutrophil interactions are necessary for NET formation in several thomboinflammatory disorders $(8,16)$, neutrophils isolated from healthy individuals (control neutrophils) were stimulated with PRP from patients with COVID-19. We found that these platelet-rich plasma-stimulated (PRP-stimulated) control neutrophils had increased levels of TF mRNA (Figure 2A) and efficiently generated TF-bearing NETs (Figure 2, B, D, and E); the corresponding NETs showed high TAT activity (Figure 2C), supporting the important role of platelets in NET-mediated COVID-19 immunothrombosis. On the other hand, although COVID-19 serum or plasma led to intracellular TF expression in control neutrophils, they were not able to trigger efficient NET formation (Supplemental Figure 1, A-C), further supporting previous studies indicating that the release of TF-expressing NETs is a double-hit phenomenon $(8,16)$. That is, PRP includes both the first hit, which induces TF expression (plasma), and the second hit, which enables NET formation and extracellular exposure of TF via NETs (platelets).

Considering the proposed role of endothelial cells in the thrombotic microangiopathy of COVID-19 $(2,17)$, we next undertook an in vitro investigation of the interplay between human aortic endothelial cells (HAECs) and the platelet/neutrophil/TF axis. Only NETs generated in vitro by control neutrophils that had been exposed to PRP from patients with COVID-19 were able to induce TF expression in HAECs that led to thrombotic potential, as indicated by TF quantitative PCR (Supplemental Figure 2A), and TAT assay in cell culture supernatants (Supplemental Figure 2B). In contrast, although HAECs treated with COVID-19-derived PRP 

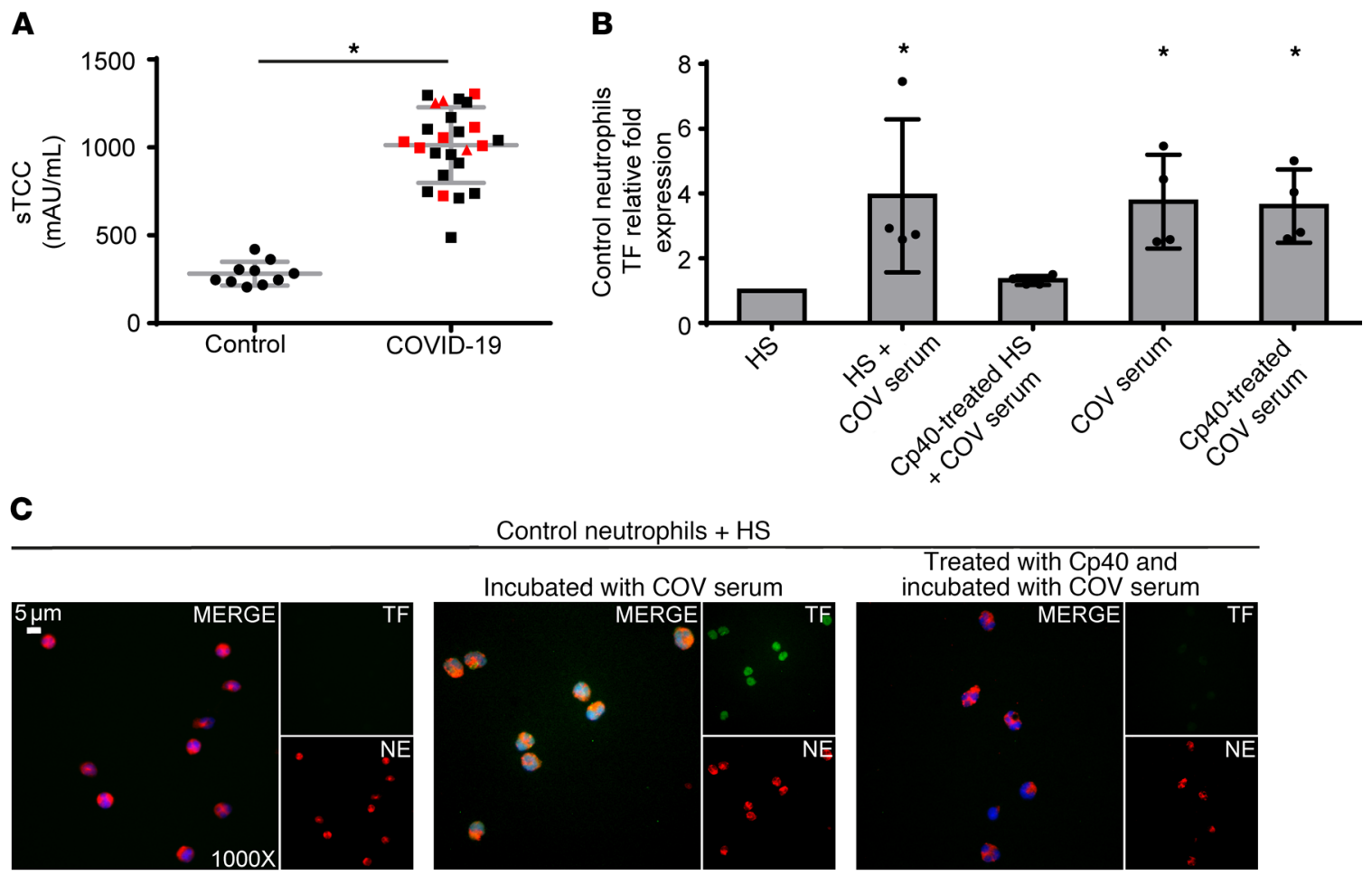

Control neutrophils + HS
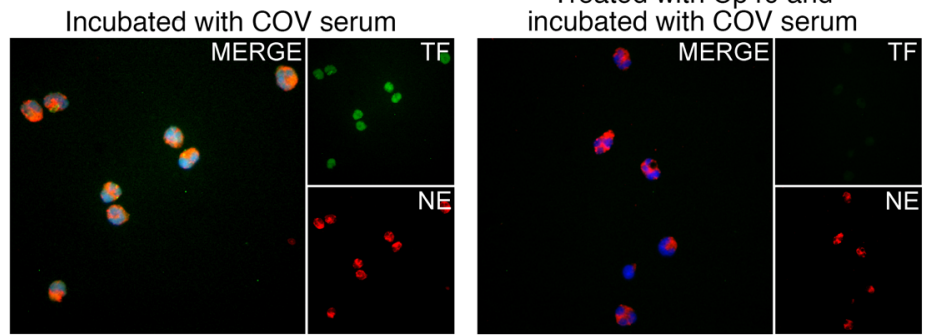

Figure 3. C3 inhibition disrupts neutrophil-driven thromboinflammation in COVID-19. (A) Soluble terminal complement complex (sTCC) levels in plasma from controls $(n=10)$ and patients with COVID-19 $(n=25)$. Red squares: severe patients; red triangles: critical patients. (B) Relative fold expression of TF mRNA in control neutrophils stimulated with serum from healthy individuals (HS), HS incubated with COVID-19 serum (COV serum), or HS treated with compstatin analog Cp40 and then COV serum, COV serum alone, or COV serum treated with Cp40. Data are from 4 independent experiments (mean \pm SD). (C) Fluorescence microscopy showing TF/NE staining in control neutrophils stimulated with HS, HS incubated with COV serum, or HS treated with Cp4O and then COV serum. A representative example of 4 independent experiments is shown. Original magnification: $\times 1000 ;$ scale bar: $5 \mu \mathrm{m}$. Blue: DAPI, green: TF, red: NE. All conditions were compared with HS alone (control). * Statistical significance at $P<0.05$. A: Student's $t$ test, B: Friedman's test. These in vitro experiments are also listed in Supplemental Table 2. Conditions of real-time RT-PCR are described in Supplemental Table 3.

alone expressed endothelial activation markers (Supplemental Figure 2C), the COVID-19 PRP was not able to stimulate TF activity in HAECs (Supplemental Figure 2, A and B). Moreover, treatment with PMA-induced NETs or PMA-induced NETs mixed with COVID-19 PRP had no effect on HAEC-derived TF expression and TAT activity (Supplemental Figure 2, A and B). In parallel, endothelial activation markers were not upregulated upon treatment of HAECs with PMA-induced NETs (Supplemental Figure 2C).

Collectively, these results suggest that COVID-19 PRPinduced NETs may amplify the TF/thrombin axis by activating endothelial cells to express TF.

Inhibition of NET-driven immunothrombosis. We next investigated the possible mechanisms governing platelet/NET/TF crosstalk. Given that activated platelets provide a catalytic surface for thrombin generation (18), we examined the role of thrombin in platelet/neutrophil interactions. Thrombin inhibition with dabigatran mitigated TF expression (Figure 2, A and F) and activity (Figure 2C), as well as NET release (Figure 2, B and F) in COVID-19 PRP-stimulated control neutrophils, indicating that thrombin contributes to platelet/neutrophil-mediated thrombogenicity. Similar effects were also obtained by blocking thrombin signaling with the FLLRN peptide against the protease-activated receptor 1 (PAR1) (Figure 2G). These findings are in line with current guidelines recommending the use of low molecular weight heparin (LMWH) in patients with COVID-19 (1). Moreover, the intracellular signaling of thrombin through PAR1 implies a potential link with inflammatory pathways (8).

Several reports have demonstrated that autophagy is tightly associated with NET formation (19). Pretreatment of control neutrophils with the autophagy inhibitor hydroxychloroquine (HCQ), which is currently being tested in patients with COVID-19, abolished NET formation in PRP-stimulated control neutrophils (Figure 2, B and $\mathrm{H}$ ), leading to a reduction in NETbound active TF, as assessed by TAT assay (Figure 2C). This finding suggests that autophagy may be involved in plateletinduced NET formation. It also suggests additional action by HCQ in COVID-19 against immunothrombosis and further supports data indicating a promising effect for HCQ in NET-related thromboinflammatory disorders $(20,21)$.

Since C5a is a key mediator of neutrophil TF expression (1315), we selectively inhibited its signaling in control neutrophils stimulated with PRP obtained from patients with COVID-19. C5a receptor (C5aR1) blockade attenuated TF expression both at the mRNA and protein levels (Figure 2, A and I, respectively); C5aRI blockade also inhibited NET release, as indicated by ELISA of MPO/DNA complex levels and immunostaining (Figure 2, B and I, respectively). Consequently, we also saw diminished TF functionality as assessed by TAT assay (Figure 2C). These findings are 


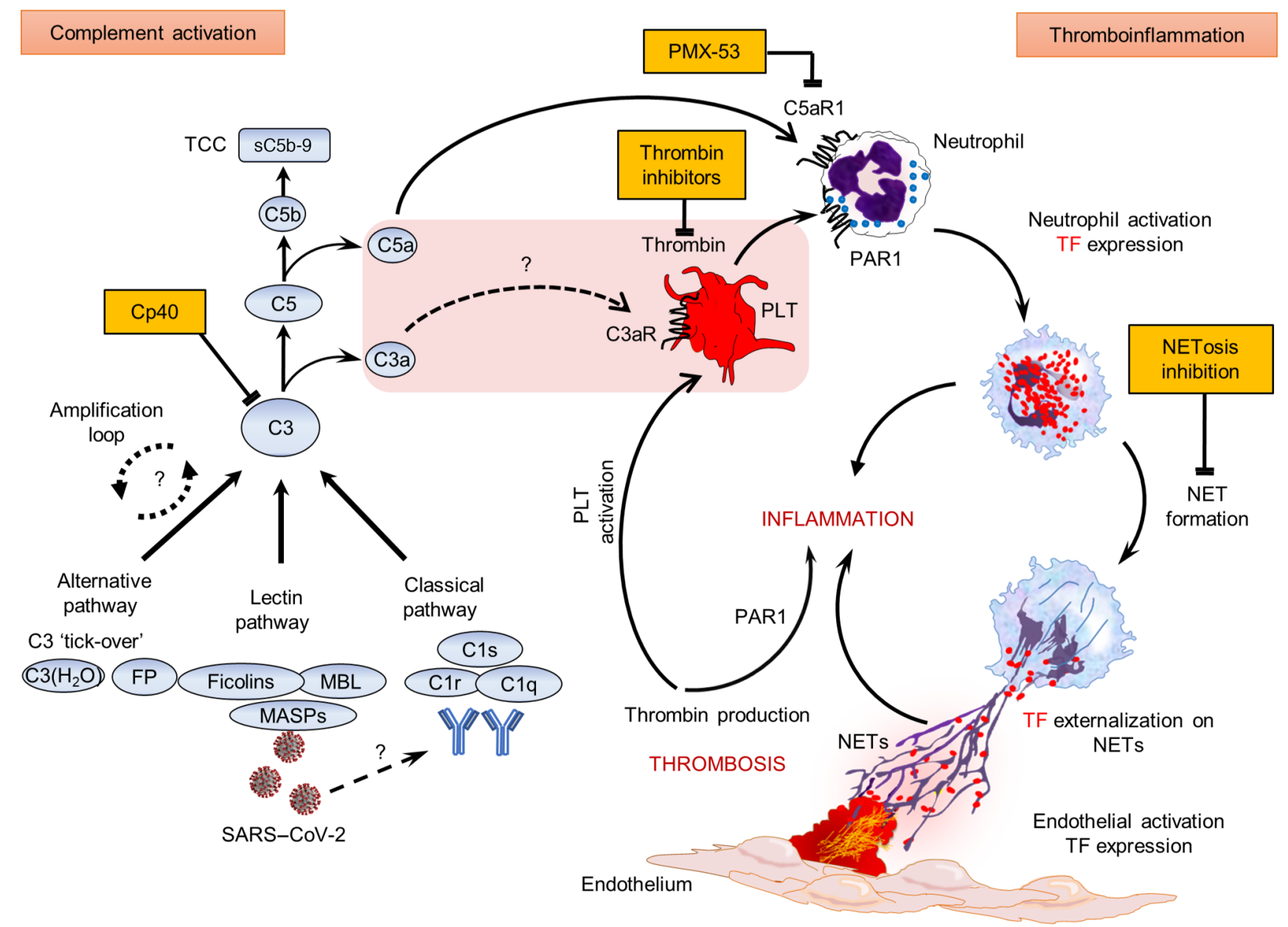

Figure 4. Proposed mechanism of COVID-19 immunothrombosis. During COVID-19, SARS-CoV-2 triggers complement activation by interacting with mannan-binding lectin (MBL) serine proteases (MASPs) or possibly through (auto)antibodies or/and immunocomplexes. C3 activation, as a point of convergence of all complement pathways, leads to C3a, C5a, and sC5b-9 (TCC) generation. Subsequently, C3a might activate platelets (PLTs), while C5a and PLT-derived thrombin induce both neutrophil TF expression and NETs carrying active TF. These thrombogenic NETs may induce endothelial cell activation toward TF expression, thus increasing their procoagulant activity. This may further amplify (e.g., via PAR1), inflammation and PLT activation, thereby fueling a complement/NET-driven vicious cycle of immunothrombosis. Complement, thrombin, and NETosis represent promising therapeutic targets. The central pink box includes components of the COVID-19 thromboinflammatory environment. Question marks and dotted lines indicate provisional pathways/connections that have not yet been investigated in COVID-19. FP, properdin; PMX-53, specific C5a receptor antagonist.

consistent with previous studies indicating a dual role for complement activation in both TF expression by neutrophils (13-15) and mechanisms related to NET formation $(22,23)$. In addition, the patients with COVID-19 exhibited considerably increased plasma levels of sC5b-9 (terminal complement complex [TCC]) (Figure 3A). Our findings are in agreement with recent evidence demonstrating markedly higher plasma levels of sC5b-9 and C5a in severely affected patients with COVID-19 and widespread complement deposition along with microvascular thrombosis in lung and skin tissue samples $(7,24,25)$.

Compstatin inhibits complement activation, disrupting neutrophil-mediated $T F$ expression. Emerging evidence from patients with COVID-19 indicates that complement activation predominantly involves the lectin and alternative complement pathways. However, the possible contribution of the classical pathway, due to immune complexes formed by natural autoantibodies or antibodies against SARS-CoV-2, cannot be ruled out $(10,25)$.
Given the central role of $\mathrm{C} 3$ as a point of convergence of all complement pathways, we hypothesized that $\mathrm{C} 3$ activation is a crucial upstream mechanism driving C5a/C5aR1-mediated responses in COVID-19.

Prompted by our findings regarding the high levels of complement activation in patients with COVID-19 (increased sC5b-9), we next examined whether COVID-19 patient serum, which represents the disease's inflammatory environment, is able to induce complement activation upstream of $\mathrm{C} 5$, leading to $\mathrm{TF}$ expression in control neutrophils. In this context, to address the role of C3 activation we developed a serum coincubation system (for details see Supplemental Methods, section 1.4.1). Serum from healthy individuals (control serum), serving as a source of nonactivated complement, was pretreated or not treated with the compstatin analog $\mathrm{Cp} 40$ (which blocks all complement pathways by targeting the central protein C3) $(10,15)$, and then coincubated with COVID-19 serum. Cp40-mediated inhibition 
of C3 significantly decreased the capacity of our serum coincubation system to induce TF expression in control neutrophils, at both the mRNA (Figure 3B) and protein levels (Figure 3C). These results indicate that $\mathrm{C} 3$ inhibition disrupts the source that triggers TF release from neutrophils, broadly preventing complement activation and impairing the thrombogenicity of the $\mathrm{TF} /$ thrombin axis in COVID-19.

COVID-19 serum contains a plurality of plausible factors that could trigger de novo $\mathrm{C} 3$ activation in the control serum; these include immune complexes due to the presence of natural autoantibodies, or cross-reactive IgM recognizing conserved epitopes of common cold coronavirus strains, or SARS-CoV-2-induced specific IgG antibodies. Triggers of C3 activation could also include virus-released pathogen-associated molecular patterns (PAMPs) such as the nucleocapsid $(\mathrm{N})$ protein that has been linked to mannan-binding lectin serine protease-2-dependent (MASP-2dependent) lectin pathway activation $(10,25)$.

The above finding is further supported by recent, promising clinical data from the compassionate use of the C3-targeted therapeutic AMY-101 (Cp40-based drug candidate, clinically developed by Amyndas Pharmaceuticals) in severely ill patients with COVID-19 with acute respiratory distress syndrome (12). AMY-101 treatment resulted in rapid normalization of inflammatory markers and improvement of respiratory function, indicating a pronounced effect of C3 inhibition on COVID-19 thromboinflammation (12). In this regard, the impact of C3 inhibition on neutrophil-derived TF expression may provide important mechanistic insights into how AMY-101 can broadly suppress the thomboinflammatory response that culminates in pronounced microvascular injury and thrombosis in patients with COVID-19. A phase II clinical trial has been launched to assess the impact of AMY-101 in patients with severe COVID-19 (NCT04395456).

Many pieces of the immunothrombosis puzzle have been identified as components of the complement/neutrophil/TF axis in various disorders $(13-15,22)$. In addition, it has recently been demonstrated that C3aR signaling can mediate platelet activation in coronary artery disease (26). The ability of Cp40 to attenuate neutrophil-driven thromboinflammation in our study could reflect a broader impact of C3 activation on neutrophil-platelet interactions that promote thrombogenic responses. In fact, C3 inhibition by compstatin disrupts CR3-dependent platelet-neutrophil complex formation, which could likely enhance thrombotic reactions relevant to COVID-19 pathology (27). Moreover, enhanced C3 expression is part of the unique host transcriptional signature in response to SARS-CoV-2 infection (28) and C3aR upregulation in the lung microvascular endothelium was recently correlated with disease severity (29), increased thrombosis, and aberrant angiogenesis in COVID-19 lung biopsies (2). The NET-lowering capacity of C3 inhibitors was recently demonstrated in patients with COVID-19 dosed with the Cp40-based clinical candidate drug AMY-101 (30). Collectively, these studies support a key role for C3 activation in driving the platelet/NETs coagulopathy axis. Here, we report that this axis is substantially involved in the pathophysiology of COVID-19 (Figure 4). We propose that COVID-19 constitutes an emerging clinical model of immunothrombosis that may help to shed light on the dark side of complement-related disorders (11). Our findings argue for the timely application of therapeutic strategies that can disrupt the vicious cycle of COVID-19 immunothrombosis by targeting complement activation or/and NET formation $(8,10-12,19,24,25)$ (Figure 4).

\section{Methods}

The subjects were 25 patients hospitalized at University Hospital of Alexandroupolis or AHEPA University Hospital of Thessaloniki, with moderate $(n=15)$, severe $(n=7)$, or critical $(n=3)$ COVID-19 $(12$ males, 13 females; mean age, $62.1 \pm 13.8$ years; Supplemental Table 1$)$. Ten healthy age- and sex-matched individuals served as controls. Neutrophils, sera, citrated plasma, and PRP were isolated from patients with COVID-19 and from controls. For in vitro stimulation, appropriate cultures of HAECs were also used.

Statistics. Statistical analyses were performed using GraphPad Prism 6 and SPSS 26. Comparisons between 2 groups were performed using Student's $t$ test (2-tailed) and Levene's test for equality of variances. For comparisons among more groups, the Friedman's 2-way ANOVA by rank was used. Bivariate correlation analysis was performed using Spearman's correlation test (at 95\% confidence intervals). $P$ value was set to 0.05 . Data are presented as mean \pm standard error of the mean.

Study approval. The study protocol was reviewed and approved by the Ethics Review Board of Alexandroupolis and AHEPA/Thessaloniki university hospitals. Subjects or their relatives provided written informed consent before study participation.

Detailed information for all methods is provided in the Supplemental Methods.

\section{Author contributions}

PS wrote the manuscript, conceived and designed experiments, and acquired and analyzed clinical data. AM and AC designed and conducted experiments, analyzed data, and contributed to writing. DCM contributed to data analysis and writing. MN, VT, CT, and TK conducted in vitro experiments. SM, PR, ES, MT, CP, IM, and GG provided clinical samples and analyzed data. JDL and KR contributed to writing, and conceived, designed, and cosupervised the study. All authors read and approved the final manuscript. The order of co-first authors was determined by relative effort in study design, clinical data acquisition, drafting of manuscript, and overall workload.

\section{Acknowledgments}

We thank Vasileios Papadopoulos for his advice in statistical analysis and Deborah McClellan for editorial assistance. This study was supported by grant T1EDK-00617 from General Secretariat for Research and Technology, Research \& Innovation Programme CYTONET. AM is cofinanced by Greece and the European Union (European Social Fund; MIS-5000432) program, implemented by the State Scholarships Foundation (Idrima Kratikon Ypotrofion).

Address correspondence to: Konstantinos Ritis, First Department of Internal Medicine, University Hospital of Alexandroupolis, Democritus University of Thrace, Alexandoupolis, 68100, Greece. Phone: 003.02551351103; Email: kritis@med.duth.gr. 
1. Connors JM, Levy JH. COVID-19 and its implications for thrombosis and anticoagulation. Blood. 2020;135(23):2033-2040.

2. Ackermann M, et al. Pulmonary vascular endothelialitis, thrombosis, and angiogenesis in covid-19. N Engl J Med. 2020;383(2):120-128.

3. Wang D, et al. Clinical characteristics of 138 hospitalized patients with 2019 novel coronavirus-infected pneumonia in Wuhan, China. JAMA. 2020;323(11):1061-1069.

4. Liu X, Zhang R, He G. Hematological findings in coronavirus disease 2019: indications of progression of disease. Ann Hematol. 2020;99(7):1421-1428.

5. Barnes BJ, et al. Targeting potential drivers of COVID-19: Neutrophil extracellular traps. J Exp Med. 2020;217(6):e20200652.

6. Fox SE, Akmatbekov A, Harbert JL, Li G, Quincy Brown J, Vander Heide RS. Pulmonary and cardiac pathology in African American patients with COVID-19: an autopsy series from New Orleans. Lancet Respir Med. 2020;8(7):681-686.

7. Magro C, et al. Complement associated microvascular injury and thrombosis in the pathogenesis of severe COVID-19 infection: A report of five cases. Transl Res. 2020;220:1-13.

8. Stakos D, Skendros P, Konstantinides S, Ritis K. Traps n' clots: NET-mediated thrombosis and related diseases. Thromb Haemost. 2020;120(3):373-383.

9. Zuo Y, et al. Neutrophil extracellular traps in COVID-19. JCI Insight. 2020;5(11):138999.

10. Risitano AM, et al. Complement as a target in COVID-19? Nat Rev Immunol. 2020;20(6):343-344.

11. Java A, et al. The complement system in COVID-19: friend and foe? JCI Insight. 2020;5(15):140711.
12. Mastaglio S, et al. The first case of COVID-19 treated with the complement $\mathrm{C} 3$ inhibitor AMY-101. Clin Immunol. 2020;215:108450.

13. Ritis K, et al. A novel C5a receptor-tissue factor cross-talk in neutrophils links innate immunity to coagulation pathways. JImmunol. 2006;177(7):4794-4802.

14. Redecha P, et al. Tissue factor: a link between $\mathrm{C} 5 \mathrm{a}$ and neutrophil activation in antiphospholipid antibody induced fetal injury. Blood. 2007;110(7):2423-2431.

15. Kourtzelis I, et al. Complement anaphylatoxin C5a contributes to hemodialysis-associated thrombosis. Blood. 2010;116(4):631-639.

16. Stakos DA, et al. Expression of functional tissue factor by neutrophil extracellular traps in culprit artery of acute myocardial infarction. Eur Heart $J$. 2015;36(22):1405-1414.

17. Varga Z, et al. Endothelial cell infection and endotheliitis in COVID-19. Lancet. 2020;395(10234):1417-1418.

18. Monroe DM, Hoffman M, Roberts HR. Platelets and thrombin generation. Arterioscler Thromb Vasc Biol. 2002;22(9):1381-1389.

19. Skendros P, Mitroulis I, Ritis K. Autophagy in neutrophils: from granulopoiesis to neutrophil extracellular traps. Front Cell Dev Biol. 2018;6:109.

20. Jung $\mathrm{H}$, et al. The protective effect of antimalarial drugs on thrombovascular events in systemic lupus erythematosus. Arthritis Rheum. 2010;62(3):863-868.

21. Bertolaccini ML, et al. Complement inhibition by hydroxychloroquine prevents placental and fetal brain abnormalities in antiphospholipid syndrome. JAutoimmun. 2016;75:30-38.

22. Huang YM, Wang H, Wang C, Chen M, Zhao MH. Promotion of hypercoagulability in antineutrophil cytoplasmic antibody-associated vasculitis by C5a-induced tissue factor-expressing microparticles and neutrophil extracellular traps. Arthritis Rheumatol. 2015;67(10):2780-2790.

23. Guglietta S, et al. Coagulation induced by C3aR-dependent NETosis drives protumorigenic neutrophils during small intestinal tumorigenesis. Nat Commun. 2016;7:11037.

24. Cugno M, et al. Complement activation in patients with COVID-19: A novel therapeutic target. J Allergy Clin Immunol. 2020;146(1):215-217.

25. Gao T, et al. Highly pathogenic coronavirus $\mathrm{N}$ protein aggravates lung injury by MASP-2-mediated complement over-activation. medRxiv. https://doi.org/10.1101/202 0.03.29.20041962. Published June 18, 2020. Accessed September 3, 2020.

26. Sauter RJ, et al. Functional relevance of the anaphylatoxin receptor $\mathrm{C} 3 \mathrm{aR}$ for platelet function and arterial thrombus formation marks an intersection point between innate immunity and thrombosis. Circulation. 2018;138(16):1720-1735.

27. Hamad OA, et al. Contact activation of C3 enables tethering between activated platelets and polymorphonuclear leukocytes via CD11b/ CD18. Thromb Haemost. 2015;114(6):1207-1217.

28. Blanco-Melo D, et al. Imbalanced host response to SARS-CoV-2 drives development of COVID-19. Cell. 2020;181(5):1036-1045.e9.

29. Yan B, et al. SARS-CoV2 drives JAK1/2-dependent local and systemic complement hyperactivation [published online June 9,2020]. Res Sq. https://doi.org/10.21203/rs.3.rs-33390/v1.

30. Mastellos DC, et al. Complement C3 vs C5 inhibition in severe COVID-19: Early clinical findings reveal differential biological efficacy. Clin Immunol. 2020;220:108598. 